\title{
Morgagnis Paré-Bild in De sedibus et causis morborum
}

\author{
Von Markwart Michler, Gießen
}

Paré und Morgagni trennen zweihundert Jahre, doch selbst, wenn sie Kinder einer Zeit gewesen wären, so hätten sie nach Herkunft und Wesen, Lebenswerk und Lebensschicksal nur wenig gemeinsam gehabt. Es wäre daher auch müßig, in De sedibus et causis morborum nach einem besonderen Verhältnis zwischen beiden Männern zu fahnden, nach Bindungen, die einige saecula zu überspringen und sich an große Tote vergangener Zeitalter anzuknüpfen vermögen; sie existierten nicht und sähen Morgagni wohl auch nicht ähnlich. Einer Untersuchung, die lediglich jenes Bild Parés nachzeichnen möchte, das uns im Werk des Paduaners entgegentritt, kann daher nur die Frage interessant sein, wie die Schulmedizin am Vorabend der Wiedereingliederung der Chirurgie zu den Leistungen eines Mannes stand, der als ungelehrter Barbier und Feldscher, als ein Stachel im Fleisch der Pariser Fakultät, die ersten Voraussetzungen für diese Entwicklung mitgeschaffen hatte. Für ein solches Vorhaben freilich mögen Werk und Autor besonders geeignet erscheinen: Einerseits hat De sedibus allein schon mit seinem grundsätzlichen Anliegen auf seine Weise noch einmal Entscheidendes zum endgültigen Aufbau einer akademischen Chirurgie beigetragen. Zum anderen ist Morgagni - obgleich Schüler des akademischen Chirurgen Valsalva und Lehrer des großen Scarpa - niemals selbst chirurgisch tätig gewesen, weil er nach eigenem Eingeständnis von Natur aus nicht für die chirurgische Praxis geschaffen war ${ }^{1}$. So mag er zugleich die erforderliche Distanz und unbefangene Neutralität besitzen, um als repräsentative Stimme seiner Zeit gelten zu dürfen.

Eine grundsätzliche Stellungnahme Morgagnis zu den Verdiensten Parés wird man in den fünf Büchern seines Werkes freilich vergeblich suchen. Er nennt ihn zur Sache, wo es ihm angebracht erscheint, bestätigt oder kritisiert seine Befunde und korrigiert auch einmal Zitate, die von anderen Schriftstellern entstellt wiedergegeben werden; darüber hinaus kein Wort. Er behandelt ihn damit jedoch kaum schlechter als die unendliche Anzahl

1 De sedibus (Epist.) L, (Artic.) 1; Ed. Patav. 1765 (P.) Tom. II, p. $230=$ Ed. Radius (R.) Tom. V, p. 40. Vgl. Verfasser, Hubers Klassiker der Medizin und der Naturwissenschaften, Band 10, Bern/Stuttgart 1967, Einführung S. 19f. 
aller übrigen Autoren, die bei ihm zu Wort kommen, und nur wer die strengen protokollarischen Gepflogenheiten kennt, die er bei der Nennung der einzelnen Namen beachtet, wird hier zum erstenmal aufmerksam: Mag es noch verständlich erscheinen, wenn er so hervorragende Männer wie ALBrecht von Haller, van Swieten oder Triller als «praestantissimus», «illustris archiater», als «amplissimus» oder «illustris vir» besonders ehren will ${ }^{2}$, so weiß er doch auch Chirurgen durch entsprechende Epitheta in die ungeschriebene Rangordnung der Wissenschaft einzufügen. Dabei verwundert es nicht, wenn er HeIsters Stellung als «medicus et chirurgus» betont, und ihn mit Beinamen wie «eruditissimus » und «experientissimus» auszeichnet $^{3}$. Doch versteht er es auch, weniger erlauchte Chirurgen anzusprechen und zitiert etwa De LA FAYE oder De LUG als «egregius» und "solers chirurgus» ${ }^{4}$. Selbst besondere Geschicklichkeit und Erfahrung in bestimmten Einzelleistungen weiß er gebührend zu würdigen und nennt beispielsweise den jüngeren Verneyus einen «chirurgus, si quis alius, in paracentesi (hydropis ascitis) exercitatissimus.$^{5}$ Nicht allein hier scheint freilich der Vielbelesene vergessen zu haben, daß Paré es war, der bei der Bauchwassersucht als erster die alte Methode bekämpfte, den Leib an mehreren Stellen mit Glüheisen zu öffnen ${ }^{6}$. Jedenfalls scheint er ihm keinen gehobenen Platz in seinem Zeremoniell zu gönnen, denn wo immer er ihn zitiert, heißt es stets nur «quod e Paraeo est» oder «Paraei historiam relego», und es ist schon viel, wenn er sich zu Sätzen herbeiläßt, wie:

${ }^{2}$ Zu Haller vgl. z.B.: De sedibus L 10; P. II $231=$ R.V 47; - zu van Swieten : XXXV 22, LIV 38, LV 23; P. II 56, 283, 294=R.III 206, V 269, V 316;- zu TrILler: L 16; P.II $232=$ R.V 52 .

${ }^{3}$ Vgl. De sedibus L 10 und VII 11; P. II 231 und I $44=$ R.V 47 und I 168.

${ }^{4} \mathrm{Zu}$ Fayus vgl. De sedibus XXIII 18; P.I $210=$ R.II 418; - zu De Luc XXX 13; P.II $20=$ R.III 61 .

5 De sedibus XXXVIII 68; P.II 105 = R.III 413. In diesem und in dem vorangehenden Artikel 67 ist Verneyus außerdem mit dem Epitheton «laudatus» versehen.

6 Vielleicht mag es für Morgagnis Schweigen bezeichnend sein, daß Paré die abdominale Paracentese gerade in der Polemik seiner Apologie gegen das alte Kauterisationsverfahren propagiert, das er hier unter jenen Operationen anführt, mit denen er begründet, warum er in seinem Werk nicht alle Operationen der Alten beschrieben habe (vgl. E.H.ACKerKNeGht, in Hubers Klassiker der Medizin und der Naturwissenschaften, Band 2, Bern/Stuttgart 1963, S. 27 f.). Morgagni selbst steht freilich auch der Abdominalpunktion bei Ascites aus verschiedenen Gründen zurückhaltend gegenüber und geht in seinen Ausführungen bis auf Celsus und Erasistratos zurück (vgl. neben dem unter Anm. 5 zitierten auch die Artikel 31 ff. in Epist. XXXVIII; P. II 91 f. = R.III $353 \mathrm{ff}$.). 
«Paraeus tamen diserte negat», oder: « et recte olim Paraeum, .., mox admonuisse.» Dabei trifft es nicht einmal zu, daß er ihn offen herabsetzt, das verbietet allein schon der konziliante Ton, in dem De sedibus generell geschrieben ist, oder gestattet es doch nur bei ganz wenigen Autoren, die ihm von Herzen zuwider sind, wie Blancard zum Beispiel, den er einen Fälscher und Plagiator nennt ${ }^{7}$. Selbst in den Registern, wo es der Sorgfältige in allen geeigneten Fällen - auch bei so einfachen Wundärzten wie Gregor Barbetti ${ }^{8}$ - niemals vergißt, dem Namen ein «laudatus » hinzuzufügen, wird dem großen Chirurgen diese Auszeichnung nicht zuteil. Er lobt ihn nicht und schilt ihn milde nur dort, wo er sich fassen läßt, Paré bleibt bei ihm immer Paré.

Will man nun die Stellen näher überprüfen, in denen Paré angeführt ist, so zeigt sich, daß die Indices von De sedibus unter seinem Namen überhaupt nur drei Verweise bieten ${ }^{9}$, während ihn doch Morgagni im Verlauf seines Werkes rund dreißigmal erwähnt oder zitiert. Diese gewiß besonders karge Anzahl von Registervermerken darf jedoch nicht zu voreiligen Schlüssen verleiten; denn auch ein BoerhaAve oder Malpighi sind nur vier- bzw. sechsmal angemerkt ${ }^{10}$, obwohl doch gerade Boerhaave im Text unvergleichlich viel häufiger zugezogen wird. Nun sind die Indices von De sedibus zwar als sorgfältig bekannt, und Morgagni selbst kritisiert sogar in seiner Praefatio die oberflächlichen und lückenhaften Register des Sepulchrets ${ }^{11}$. Dennoch sind auch seine eigenen Indices keine vollständigen Generalregister im heutigen Sinn, sie bleiben im Grunde Auswahlregister, zum mindesten was die Personennamen betrifft. Zweifellos sind sie mit Bedacht und Sachverstand angelegt und befolgen im einzelnen feste Regeln. Doch Sach- und Personenverweise korrespondieren keineswegs miteinander, und was unter dem Sachwort Erwähnung findet, wird häufig - aber durchaus nicht regelmäßig - unter dem zugehörigen Autorennamen nicht mehr angeführt. So ist denn auch hier der Verdacht geweckt, gewisse protokollarische Erwä-

7 De sedibus XXXII 1; P.II $32=$ R.III 108;- siehe auch XXII 2 ; P.I 194= R. II 351; XXIII 1 und 10; P.I 203 und $206=$ R.II 389 und $402 ;-$ XXXIV 1; P. II $40=$ R. III $141 \mathrm{f}$.

8 Vgl. P.I, Index quartus, p. LXI s.v.= R. VI, Index secundus, p. 202 s.v.

9 Vgl. P.I, Index quartus, p. LXX s.v. = R. VI, Index secundus, p. 315 s.v.

10 Vgl. P.I, Index quartus, p. LIII s.v. BoERhaAvius = R. VI, Index secundus, p. 204 s.v. (Die Zitatnachweise sind in beiden Editionen in unterschiedlicher Reihenfolge aufgeführt.)-P.I, Index quartus, p. LXVII s. v. MALPIGHI = R. VI, Index secundus, p. 294 s. v.

11 De sedibus, praefatio 6; P.I, p.X = R.I, p. XLIV f. 
gungen könnten die Redaktion der Personenverweise über Gebühr beeinflußt und die positive oder negative Bewertung einzelner Zitate im Text könnte - je nach Gunst - auch über die Art ihrer Aufnahme in die Register entschieden haben. Unter diesen Umständen verwundert es jedenfalls nicht, wenn Männer wie Valsalva, Haller oder Heister mit rund 60,80 und 45 Stellenvermerken vollständig oder doch fast vollständig erfaßt und auch ein Mediavia oder Pasta mit 45 und 13 Notizen gebührend berücksichtigt sind ${ }^{12}$. Selbst Hippokrates schneidet mit 21 Verweisen recht gut $\mathrm{ab}^{13}$, Paré aber steht - wenn auch sicher nicht als einziger - am unteresten Ende dieser Skala. Solche Beckmesserei mag Schelte verdienen, aber nur sie kann zeigen, daß in der Zahl und Art der Stellenauswahl tatsächlich ein Kriterium für die Gesinnung gegeben ist, die Morgagni gegen Paré unausgesprochen hegte.

Will man dennoch ein falsches Bild vermeiden, dann wird man bei der Überprüfung der einzelnen Zitate die wenigen Textstellen besser unberücksichtigt lassen, die entweder ohne Zitatnachweis gegeben werden ${ }^{14}$ oder Wiederholungen darstellen ${ }^{15}$. Auch jene größere Anzahl wird man zunächst zurückzustellen haben, die indirekt - meist über BonETs Sepulchretum zitiert ist ${ }^{16}$. Bis auf ein Zitat ${ }^{17}$ sind in diesen beiden Gruppen bereits auch

12 Vgl. P.I, Index quartus, p. LXXIX s.v. VALsalva = R. VI, Index secundus, p. 370 f. s.v. (Die Zitatnachweise sind in beiden Editionen in verschiedener Reihenfolge aufgeführt.) P. I, Index quartus, p. LXI s.v. Haller, p. LXI f. s.v. Heister, p. LXVII s.v. Mediavia, p. LXX s.v.PASta = R. VI, Index secundus, p. 262 f. s.v.,p. 263 s.v.,p. 293 s.v.,p. 316 s.v.

13 Vgl. P.I, Index quartus, p. LXII s.v. = R. VI, Index secundus, p. 266 f. (Die Zitatnachweise sind in beiden Editionen in unterschiedlicher Reihenfolge aufgeführt; ein falscher Nachweis bei R. [statt: XX 7 muß es heißen: XXVIII 7].)

14 De sedibus XIX 45; P. I $162=$ R.II 219. - L 30; P.II $236=$ R.V 67. - LVI 3; P. II $297=$ R.V $326 \mathrm{f}$. (Der Zitatnachweis erfolgte hier freilich bereits im vorangehenden Art. LVI 2.)

15 De sedibus XXVI 27; P.I $242=$ R. II 555 Wiederholung von: XXIV 36; P. I $228=$ R.II 494 ff. - XXXIII 7; P. II $37=$ R.III 130 Wiederholung von: XXXIII 3; P.II $36=$ R. III 126. - LVI 9; P.II $299=\mathrm{R}$. V 335 Wiederholung von: LVI 2 ; P.II $296 \mathrm{f} .=\mathrm{R}$. V $325 \mathrm{f}$.

${ }^{16}$ De sedibus V 13; P.I $36=$ R.I 133. - XXIV 35/36; P.I 227 f. = R.II 493 ff. - XLI 17; P.II $135=$ R.IV 125. - LIII 1; P.II $268=$ R.V 205. - LVI 34; P.II $306=$ R.V 363 f. LIX 4; P.II $321=$ R.V 427 (zitiert aus: TH.Bonet, Sepulchretum sive Anatomia practica, Editio altera von J. J.MANGET, Genf 1700). - VIII 26; P.I 54=R. I 210 f. (zitiert aus: J.SchenK von Grafenberg, Observationum medicarum rararum, novarum, admirabilium et monstrosarum volumen, Basel/Freiburg 1584-1597). - XXXIX 3/4; P. II $107=$ R.IV 5 (zitiert aus: Th. WharToN, Adenographia sive glandularum totius corporis descriptio, Amsterdam 1659).

17 De sedibus LIV 12; P. II $278=$ R. V 246. 
all jene Stellen enthalten, in denen Paré lediglich innerhalb einer längeren Reihe von Autoren erwähnt ist und die auch unter den restlichen Autorennamen im Register nicht eigens aufgeführt sind.

Unter einer so strengen Auslese bleibt dann noch immer ein Rest von zwölf direkten Zitaten übrig, und ihr Inhalt ist einer näheren Betrachtung wert: In Epistel XII erwähnt Morgagni die von der Antike her bekannte Operation eines Hydrocephalus internus, bei der die zwischen den Schädelnähten hervortretende Geschwulst durch einen Schnitt eröffnet wurde ${ }^{18}$. In der Ablehnung dieses Eingriffs, der offensichtlich seit der Renaissance von neuem geübt wurde, stützt er sich auf Parés Erfahrungen, nach denen noch niemand durch diese Maßnahme gesund geworden sei ${ }^{19}$. Auch für die damals noch umstrittene Tatsache, daß ein kongenitaler Hydrocephalus mit einer Verkümmerung des Cerebrum einhergehen kann, fußt er auf Beobachtungen Parés ${ }^{20}$. Zweifellos haben beide Zitate wesentlich zu Morgagnis Urteilsbildung beigetragen, doch im Register finden sie keine Berücksichtigung.

Auch bei der Behandlung der Aortenaneurysmen im XVII. Brief erwähnt der Paduaner den Chirurgen im Rahmen der pathologischen Differentialdiagnostik mit seiner Beobachtung einer faustgroßen Gefäßerweiterung an der Vena pulmonaris ${ }^{21}$. «Paré bezeichnet sie», so schreibt er wörtlich weiter, «nicht als Varize, sondern als Aneurysma, und er konnte dies in der Tat aus demselben Grund tun, aus dem er, wie man zu seiner Zeit gewohnt war, jene Vene als ,arteria venosa' bezeichnete.» Seine Worte beziehen sich direkt auf das 32. Kapitel des VI. Buches bei Paré ${ }^{22}$, und es ließe sich über den fehlenden Registervermerk hinweggehen, wenn nicht Morgagnis Ausführungen an dieser Stelle lediglich die Exposition zu weiteren Erörterungen im XXIV. Brief bildeten. Dort kommt er noch einmal auf die Aneurysmen der großen Gefäße zurück und zitiert zwei Observationes von BaIllou und abermals von Paré indirekt über das Sepulchretum. Paré

18 De sedibus XII 13; P. I $81=$ R. I 324.

19 PARÉ, Opera chirurgica, lib. VII, c. 1. Es wurde die lateinische Ausgabe, Frankfurt am Main 1594, herangezogen, die offensichtlich auch BoNET und MANGET für das Sepulchretum benutzt haben; ebenda p. 227.

${ }_{20}$ De sedibus XII 13; P. I 82 = R.I 325 f. - Sepulchretum, lib. I, sect. XVI, obs. 12; Tom. I, p. 384. - PARÉ, Opera chirurgica, ibidem.

${ }^{21}$ De sedibus XVII 3; P.I $132=$ R. II 90.

22 Vgl. Paré, Opera chirurgica, lib. VI, c. 32 ; p. 224 f. (falsche Kapitelbezeichnung in der benutzten lateinischen Edition, die hier in der Zählung auf c. 28 zurückspringt). 
berichtet darin ebenfalls von einer bis auf Faustgröße ausgedehnten «arteria venosa », deren Innenwand knöchern verändert war, und eine nähere Überprüfung zeigt, daß es sich hier um die nämliche Beobachtung handelt, die Morgagni bereits im XVII. Brief direkt aus Paré zitiert und für die das Sepulchretum die fehlerhafte Kapitelbezeichnung der lateinischen ParéAusgabe übernommen hatte ${ }^{23}$.

Offensichtlich bemerkt auch Morgagni den Irrtum nicht, denn ahnungslos geht er im folgenden Artikel nochmals auf Parés Beobachtung ein: «Ich sprach aber von Aneurysmen», so schreibt er, «und zählte auch Parés Observatio dazu, erstens weil er hier dieselbe Bezeichnung, nämlich Aneurysma, für eine ,arteria venosa ${ }^{6}$ gebraucht, zweitens aber auch, weil es mir zweifelhaft erscheint, ob er nicht vielleicht aus Nachlässigkeit so geschrieben hat, während er doch eigentlich, vena arteriosa" schreiben wollte; d. h. ,arteria pulmonaris ${ }^{6} .{ }^{24}$ Ungenauigkeit ist sonst Morgagnis Sache nicht, und so hegt er auch gegen diese Annahme noch Zweifel, denn Paré berichtet ja, die «tunica interna» des Gefäßes sei knöchern verändert gewesen, während er doch an anderer Stelle sagt, die Venen bestünden überhaupt nur aus einer einzigen «tunica», und lediglich die Arterien besäßen deren zwei. Nur diese letzte Behauptung zitiert Morgagni direkt aus Parés Werk ${ }^{25}$, für die vorangehenden Ausführungen bleibt es bei dem indirekten Zitat aus dem Sepulchretum. Morgagni zitiert also hier ein und dieselbe Observatio einmal im XVII. Brief direkt, ohne an ihr Anstoß zu nehmen, und ein zweites Mal indirekt im XXIV. Brief, wo er die Gelegenheit zu einem abwertenden Tadel nicht ungenutzt vorübergehen läßt, obwohl er selbst nicht einmal bemerkt, daß er in beiden Fällen den nämlichen Text vor sich hat. Mag ein solches Verhalten bereits über seine Objektivität in dieser Sache Auskunft geben, so dürfte es erst recht bezeichnend sein, daß er im Register nur das zweite, vermeintlich neue, indirekt übernommene Zitat aus Epistel XXIV vermerkt, mit seiner negativen Kritik, sei es nun an Parés Sorgfalt, sei es an seinen Kenntnissen in der anatomischen Nomenklatur. Noch einmal hatte er zuvor schon im XVIII. Brief den großen Chirurgen zum Thema

${ }^{23}$ De sedibus XXIV 35; P.I 227 f. = R.II 493 f. - Sepulchretum, lib. II, sect. IX, obs. 9;

Tom. I p. 848; das Sepulchretum übernimmt an dieser Stelle für Paré stillschweigend die falsche Kapitelbezeichnung (c. 28) der lateinischen Edition. In Wirklichkeit handelt es sich um das nämliche Zitat, das Morgagni bereits XVII 3 herangezogen hatte (vgl. Anm. 21 und 22).

${ }_{24}$ De sedibus XXIV 36; P.I $228=$ R.II 494. f.

${ }^{25}$ Vgl. Paré, Opera chirurgica, lib. II, c. 10; p. 74. 
der Aortenaneurysmen zu Wort kommen lassen mit seiner Mahnung, sich in der Diagnose nicht auf die Pulsation als ein sicheres Zeichen zu verlassen: Denn bei übermäßig großen Aneurysmen sei keinerlei Puls wahrzunehmen, da das Blut in Thromben zusammengebacken und geronnen sei ${ }^{26}$. Doch auch dieser wichtige diagnostische Hinweis, der gleichfalls dem besagten 32. Kapitel aus Parés VI. Buch enstammt, ist im Register nicht aufgeführt.

Unter diesen Umständen wird auch die Art interessant, in der er im XXIV. Brief Baillou und Paré gemeinsam zitiert: Spricht er doch von zwei Observationes, «quae ex duobus celeberrimis Parisiensibus auctoribus in Sepulchretum relatae sunt». Hier zum ersten und einzigen Mal in den gesamten Büchern von De sedibus wird Paré das Epitheton «celeberrimus» zuteil, doch der forlianische Nobilis scheint auch dann noch genau aufs Protokoll zu achten und schreibt an dieser Stelle von «sehr berühmten Autoren », nicht jedoch, wie sonst so häufig, von sehr berühmten Ärzten. So mag denn das «celeberrimus» eher dem auch an anderer Stelle ausgezeichneten Baillou als Paré gegolten haben.

Noch einmal bezieht sich Morgagni direkt auf dieses Kapitel, als er in Epistel XXVII auf die steinharten Plâcques der atheromatös veränderten Aorta zu sprechen kommt. Seiner Meinung nach beschwören sie die Gefahr einer Ruptur dadurch herauf, daß ihre scharfen und unregelmäßigen Ränder die Gefäßwand erodieren und exulcerieren. Wenngleich seine Ansichten noch stark vom iatromechanischen Denken abhängig sind, so kritisiert er Paré doch zweifellos zu Recht, der glaubte, diese knochenartigen Schuppen seien von der Natur als Schutzwall aufgebaut, um den Anprall des Blutes zu hemmen und so die Rupturgefahr zu mindern ${ }^{27}$. Hier nun bedient sich der Paduaner einer ungewöhnlich scharfen Polemik, die mit den Worten beginnt: «So stark irrte Paré von der Wahrheit ab, ...», und in deren Folge er dessen Ansichten halb ironisch abwertet. Sollte es wirklich nur Zufall sein, daß Morgagni jenes ominöse 32. Kapitel ausgerechnet an dieser Stelle mit ihren herabsetzenden Randbemerkungen als einziges direktes Zitat unter Paré in den Index aufgenommen hat? Denn der dritte noch ausstehende Registerverweis unter Paraeus betrifft einen Abschnitt aus

${ }^{26}$ De sedibus XVIII 20; P. I $146=$ R. II 148. Auch hier bezieht sich MorgagnI direkt auf cap. 32 des VI. Buches bei PARÉ, das in der lateinischen Edition die falsche Kapitelbezeichnung (28) trägt.

${ }^{27}$ De sedibus XXVII 24; P. I $253=$ R. II 601; - PARÉ, Opera chirurgica, ibidem. 
den Observationes des Schenk von Grafenberg ${ }^{28}$, der vom Untertauchen wasserscheue1 Tollwutkranker berichtet. Es handelt sich also um jene sinnlose Maßnahme, die bereits Celsus bei der Hydrophobia empfohlen hatte ${ }^{29}$, und Schenk zitierte hier zu dieser Frage eine Reihe anderer Autoren, darunter Paré. Gerade dessen Ansicht aber kommt Morgagni äußerst gelegen, da sie ihm die Nutzlosigkeit dieser Methode bestätigt, und so verweist er in der weiteren Folge seiner Ausführungen sogar auf den Ursprungsort in seinen Opera chirurgica ${ }^{30}$. Freilich gibt Paré dem Paduaner auch hier Gelegenheit zur Kritik, denn er erwähnt in diesem Zusammenhang die falsch aus Aetros übernommene Historie über einen Philosophen, der, von der Rabies befallen, gebadet und unerschrocken Wasser getrunken habe, und auf diese Weise gesund geworden sei ${ }^{31}$. Wer hier noch Zweifel hegt, ob das richtige klinische Urteil oder die falsche Anekdote Morgagni zur Aufnahme ins Register veranlaßte, dem wird Gewißheit, wenn er das böse Schlagwort: «Paraei hallucinationes» liest, unter dem sich dieses und das vorangehende Zitat im Index zusammengefaßt finden.

Die nämliche Gesinnung wird auch in den übrigen Zitaten deutlich, die nicht im Register berücksichtigt sind:

Die Ursachen des Analprolaps waren zu jener Zeit noch nicht geklärt, und so bestanden eine Reihe verschiedener ätiologischer Theorien nebeneinander. Als zwei Vertreter der These, eine Erschlaffung des Schließmuskels sei für dieses Leiden verantwortlich, erwähnt Morgagni dabei seinen Landsmann Mercurialis gemeinsam mit Paré in der bezeichnenden Form: «eruditissimus civis meus Hieronymus Mercurialis et Ambrosius Paraeus ». ${ }^{32}$ Nur vorsichtig distanziert er sich hier von deren Ansicht mit dem Hinweis auf Krankheitsfälle, in denen der Sphincter ani nachweisbar intakt war. Bei beiden Männern findet sich kein Registervermerk über diese These, die der Paduaner zurückgewiesen hatte. Indessen ist Mercurialis in der vorausgehenden Epistel mit dem nämlichen Abschnitt seines Buches Über die

${ }^{28}$ De sedibus VIII 26; P. I 54=R. I 210 f. - J. Schenk von Grafenberg, Observationes, lib. VII, p. 22.

${ }^{29}$ Celsus, De medicina, lib. V, c. 27, 2 C; Ed.Loeb II, p.112.

30 PARÉ, Opera chirurgica, lib. XX, c. 14; p. 584.

31 Von Morgagni richtiggestellt anhand von Aetios, Tetrabibl. II, serm. 2, c. 24; Ed. J. Cornarius, Basel 1549, p. 284= CMg VIII 2 (lib. VI c. 24) p. 164.

${ }^{32}$ De sedibus XXXIII 3; P.II 36=R.III 126. - H.MercurIalis, De morbis puerorum tractat., Venedig 1601, lib. I, c.10; f. 45 v. f. - Paré, Opera chirurgica, lib. VII, c. 18; p. 246. 
Kinderkrankheiten schon einmal im positiven Sinn zur Atresia ani zitiert worden, und Paré widerfährt gleichfalls eine zustimmende Würdigung wenige Artikel später zur Therapie des Analprolaps ${ }^{33}$. Doch nur Mercurialis taucht mit seinem positiven Zitat im Index auf ${ }^{34}$, Paré wird auch hier stillschweigend übergangen, und so dürfte es kaum zweifelhaft sein, wen Morgagni mit seinem Schweigen bei dem gemeinsamen Zitat schonen wollte. Noch deutlicher dürfte diese Verfahrensweise im XLII. Brief zum Ausdruck kommen, wo er von einem Harnkonkrement berichtet, das sich um eine als Fremdkörper in die Blase eingebrachte Nadel gebildet hatte ${ }^{35}$. Bei der Besprechung des Falles erwähnt er ähnliche Beobachtungen von Paré, Mead und Cheselden ${ }^{36}$; Mead und Cheselden werden im Register aufgeführt ${ }^{37}$, - Paré jedoch nicht.

Im LI. Brief geht Morgagni anhand von infizierten Kopfverletzungen mit sekundärer Abszeßbildung in den Thorax- und Abdominalorganen auf die Vorbedingungen und den Mechanismus der Eiterübertragung innerhalb des menschlichen Organismus ein ${ }^{38}$. Auch in dieser so bedeutsamen Frage stützt er sich abermals auf Paré, der an entsprechenden Krankheitsfällen zeigen konnte, da $\beta$ es bei Lungen- und Gelenkempyemen nur dann zum eitrigen Befall der Nieren oder Abdominalorgane kommt, wenn der Eiter am Primärherd keinen $A b f l u ß$ nach außen hat ${ }^{39}$. Überhaupt muß die Frage nach den Bedingungen der eitrigen Metastasierung Morgagni mehrfach beschäftigt haben, denn schon im ersten Buch seines Werkes ist er bei der tuberkulösen Meningitis auf dieses Problem näher eingegangen ${ }^{40}$. Auch hier fußt er auf einer im Sepulchretum angeführten Observatio Parés, bei der sich gleichzeitig Eiter in Lungen und Meningen gefunden hatte ${ }^{41}$. Selbst für diese bei-

${ }_{33}$ De sedibus XXXII 3; P.II $32=$ R. III 109 f. - H.Mercurialis, loc. cit. c. 9; f. 45 a und De sedibus XXXIII 7; P. II 37=R. III 130. - PARÉ, ibidem.

${ }^{34}$ Vgl. P.I, Index quartus, p. LXVI s.v. Mercurialis = R. VI, Index secundus, p. 297.

${ }^{35}$ De sedibus XLII 28; P. II $147 \mathrm{f}$. $=$ R. IV $174 \mathrm{ff}$.

36 ibidem 29; P.II $148=$ R.IV 176.

37 Vgl. P.I, Index quartus, p. LXVII s.v. Mead und p. LV s.v. Cheseldenus (bei beiden falscher Verweis auf art. 28 statt 29) = R.VI, Index secundus, p. 296 s.v. MEAD und p. 226 s.v. Cheseldenus (lediglich bei Mead falscher Verweis auf art. 28 statt 29).

38 De sedibus LI 23; P.II $249=$ R.V 123.

39 Paré, Opera chirurgica, lib. XVI, c. 49, und lib. X, c. 12 ; p. 500 und 346 f.

40 De sedibus V 13; P. I 36=R. I 133.

${ }^{41}$ Sepulchretum, lib. I, sect. I., obs. 40; Tom. I, p. 23. Das Sepulchretum gibt hier für Paré einen falschen Stellenverweis, der sich nicht verifizieren läßt. Auch MorgagnI scheint dies schon vergeblich versucht zu haben, jedenfalls hat er seinem Hinweis ein vorsichtiges «ut videtur» hinzugefügt. 
den wichtigen Befunde fehlt jedoch im Register ein Verweis unter dessen Namen.

Noch einmal bezieht sich der Paduaner auf den großen Chirurgen in der Differentialdiagnostik zwischen Hüftverrenkung und Schenkelfraktur ${ }^{42}$ : Paré habe nämlich aufrichtig bekannt, daß es mitunter selbst vorsichtigen Wundärzten und bei der Behandlung einer älteren Frau auch ihm selbst unterlaufen sei, einen Schenkelkopfabriß oder Schenkelhalsbruch für eine Luxation gehalten zu haben. Er sei dabei vom Trochanter maior getäuscht worden, den er für den Hüftkopf gehalten habe, bis er am zweiten Tage bei einem weiteren Repositionsversuch durch das Geräusch der Bruchstücke an eine Fraktur gemahnt worden sei. Zutreffend schreibt Morgagni im Anschlu $\beta$, soweit er wisse, sei Paré der erste gewesen, der diesen allgemeinen Irrtum aufgedeckt habe, und so kann er denn auch diese wichtige Entdeckung, die eine jahrhundertealte Irrlehre richtigstellt, nicht gänzlich im Index übergehen. Doch selbst bei diesem Zitat, das zugleich ein echtes Bekenntnis im Sinne der alten hippokratischen Ethik enthält, läßt er Paré nicht die Ehre und vermerkt lediglich unter dem Sachwort «femur» den Hinweis: «femoris fracturam posse luxationem videri, quis primus animadverterit ${ }^{43}$. Übergangen ist endlich auch seineheutenoch gültige Mahnung, eine Luxation nicht alt werden zu lassen, sondern sofort zu reponieren, die in einem der folgenden Artikel über zehn Zeilen lang wörtlich zitiert ist ${ }^{44}$.

Die Ergebnisse der Untersuchung lassen sich an diesem Punkt in wenigen Worten zusammenfassen: Von zwölf direkten Zitaten Parés ist als einziges unter seinem Namen im Register ausgerechnet jene Stelle aufgeführt, wo Morgagni die Ansichten des Chirurgen einer heftigen Kritik unterzieht. Die übrigen elf dagegen, die wichtige diagnostische Hinweise, entscheidende Beobachtungen und bedeutende Entdeckungen enthalten, sind übergangen. Die beiden restlichen Registervermerke verweisen hingegen auf zwei indirekte Zitate, die ebenfalls mit einer negativen Stellungnahme verbunden sind, während auch die übrigen indirekten Zitate - das sei hier nachgetragen - von zustimmenden oder bestätigenden Äußerungen Morgagnis begleitet werden. Gewiß, darf man Indexordnungen im Einzelfall nicht allzu ernst nehmen, Zufälle und Versehen sind auch in den Registern von $D e$

42 De sedibus LVI 2; P. I 296 f. = R.V 325. - PARÉ, Opera chirurgica, lib. XIV, c. 21 ; p. 416.

43 Vgl. P. I, Index quartus, p. LX s.v. femoris = R. VI, Index secundus, p. 254 s.v.

44 De sedibus LVI 6; P. II $298=$ R. V 333. - PARÉ, Opera chirurgica, lib. XVII, c. 22; p. 529; allerdings mit falscher ätiologischer Deutung, die sich freilich auch noch MoRGAGNI zu eigen macht. 
sedibus hin und wieder im Spiel. Die Konstanz jedoch, mit der sich solche Befunde bei Paré wiederholen, und das gehässige Wort von den Paréschen Halluzinationen, dürften in Verbindung mit den eingangs geschilderten Usancen des gesamten Werkes an der verschleierten Absicht keinerlei Zweifel lassen. Doch selbst bei dem feingesponnenen Geist Morgagnis ist diese Absicht doch wiederum nicht so fein gesponnen, daß sie sich nicht ablesen ließe und vielleicht sogar ablesen lassen sollte. Sie gilt nicht Paré allein, sondern offenbar allen angesehenen Männern aus dem alten Chirurgenstande, und eine gleichgerichtete Untersuchung über Hildanus, Dionis oder Littre würde zu demselben Ergebnis führen. Auch sie sind ausschließlich mit negativen Stellen im Register aufgeführt und müssen sich wie Paré selbst hier noch Tadel gefallen lassen ${ }^{45}$. Die Wundärzte seines Heimatlandes nimmt er freilich von einer solchen Behandlung bis zu einem gewissen Grade aus. Doch wie Barbetti bereits zeigte ${ }^{46}$, hat es eher den Anschein, daß er nicht so sehr den bedeutenden unter ihnen seine Reverenz erweisen, als all jene seiner Anerkennung versichern wollte, die ihre Ausbildung unter der Obhut der italienischen Universitätsmedizin genossen hatten. Immerhin hält er auch zu ihnen eine gewisse Distanz, und es läßt sich die offensichtliche Befriedigung gar nicht verkennen, mit der er zum Beispiel von einem Streit um die richtige Diagnose zwischen seinen Studenten und einigen Wundärzten berichtet, wie sich die «seniores chirurgi» von ihrer vorgefaßten Meinung nicht abbringen ließen und die Obduktion schließlich doch die Ansicht der jungen Studiosi bestätigte ${ }^{47}$. Für die französischen Chirurgen aber - und sie dürften um diese Zeit vor allem zählen - ist bei Morgagni deutlich ein Einschnitt mit der Umwandlung des alten Collège St. Côme in die Académie Royale gesetzt, und bereits Petit, der diese Hebung des Chirurgenstandes noch tätig miterlebte, findet sich als «clarissimus chirurgus » apostrophiert ${ }^{48}$ und darf im Register sogar ein «laudatus» hinter seinem Namen führen ${ }^{49}$.

45 Vgl. P.I, Index quartus, p. LVIII s.v. Dionisius; p. LXII s.v. Hildanus; p. LXVI s.v. Littrius = R.VI, Index secundus, p. 246 s.v. Dionisius; p. 266 s.v. Hildanus; p. 292 s.v. Littrius.

46 Siehe Anm. 8.

47 Vgl. De sedibus LVI 9; P. II $299=$ R. V 335.

${ }^{48}$ Z.B. De sedibus XVI 13; P. I 119=R.II 37; - als «experientissimus» findet PETIT sich bezeichnet XVII 29; P.I $138=$ R.II 118.

49 Vgl. P.I, Index quartus, p. LXXI s.v. Petitus Jo. Ludovicus = R. VI, Index secundus, p. 322 s.v. 
Nach diesen Ergebnissen läßt sich nur schwer die Frage unterdrücken, ob eine solche Gesinnung auch vor einer offenen Herabsetzung von Parés Leistungen nicht haltmachte und sich zu Urteilen hinreißen ließ, die einer deutlichen Verfälschung der zugrundeliegenden Tatsachen gleichkämen. Ein letztes, bisher ausgespartes Zitat könnte zunächst diesen Anschein erwecken, denn auch mit ihm war zweifellos eine Schmälerung von Parés Verdiensten verbunden, die dem Paduaner nur allzu gelegen kommen mußte.

In dem Abschnitt über die Behandlung der «tumores sanguinei» an den Extremitäten erörtert er deren operative Beseitigung durch Resektion und Unterbindung der Blutgefäße und erwähnt hier Parés unvergängliches Verdienst, die Gefäßligatur bei der Gliedmaßenamputation ${ }^{50}$.

Er beginnt seine Ausführungen sogleich mit einem Fall aus der Veterinärmedizin: «Ein angesehener Arzt aus Verona erzählte mir und anderen zufällig mit mir anwesenden Gelehrten zu Venedig anno 1707 folgendes: In seiner Vaterstadt seien kürzlich einem edlen Pferd beide hinteren Schenkel bis zum Kniegelenk stark angeschwollen von einem ,tumor ${ }^{61}$, der zwar wäßrig, aber hart war und mit Schmerzen einherging. Als alle anderen Heilmittel überhaupt nichts nützten, sei ein Tierarzt erschienen, der sich anheischig machte, er werde das Tier dadurch heilen, daß er das Fell etwas oberhalb der Schwellung inzidiere und die Vene, die dort nicht gerade dünn ist, anschneide. Danach werde er sie mit zwei Fäden kräftig zusammenziehen und direkt zwischen beiden Ligaturen durchtrennen. Zunächst sei von allen widersprochen worden, denen der Blutkreislauf bekannt gewesen sei, gleich als ob diese Maßnahme nicht nur nichts nützen, sondern in höchstem Grade schaden würde. Als jener Tierarzt jedoch von sieben oder acht Pferden mit der nämlichen Krankheit berichtete - zwei davon in derselben Stadt, die von ihm auf diese Weise geheilt worden seien, und sich alsbald auf entsprechende Erkundigungen und Nachforschungen die Wahrheit seiner Worte bestätigte, gab man ihm die Erlaubnis. Jener Arzt aber, der es mir erzählte, hat als Augenzeuge mit angesehen, daß der Eingriff so vorgenommen wurde, wie er vorgesehen war. Demnach seien die Schwellungen von diesem Zeitpunkt an - sogar schon in den ersten Tagen - ständig kleiner geworden, bis sie um den zwanzigsten Tag gänzlich verschwunden seien.»

${ }^{50}$ De sedibus L 54; P. II 241 f. = R. V 89 ff. - PARÉ, Opera chirurgica, lib. XI, c. 24; p. 373; vgl. auch derselbe, Dix livres de la chirurgie etc., Paris 1564, $\mathrm{f}$. 111 a ff.

51 «Tumor» hier im Sinn der Zeit ganz allgemein für «Schwellung» gebraucht; in der Folge daher auch mit «Schwellung» übersetzt. 
Diese erfolgreiche Operation am Pferd nimmt Morgagni zum Ausgangspunkt einer längeren Diskussion: Nur kurz streift er dabei GulıELminis Ansicht, gemeinsam mit der Vene sei vielleicht ein Nerv, der zu dem erkrankten Teil gehörte, durchschnitten und so der Schmerz und damit zugleich der Reiz behoben worden, der die Schwellung unterhalten habe. Doch Morgagni geht auf diesen offensichtlich von der Irritationslehre inspirierten Erklärungsversuch nicht näher ein, sondern steuert unmittelbar auf den Kern seiner Ausführungen hin: «Wie dem auch sei», so fährt er fort, «ich wollte Ihnen diese Kur nicht verschweigen, denn es hat den Ärzten niemals geschadet zu wissen, was den Tierärzten zum Erfolg verholfen hat. Da es bei den dumpfen Tieren eher freisteht, ein Wagnis einzugehen, wurde von ihnen vielleicht schon früher mehr versucht und praktiziert, als man an Menschen vornehmen würde.» Und in diesem Zusammenhang erinnert er an Petrus Crescenntius, der «vor reichlich vierhundert Jahren über die Agricultur schrieb und gewisse Dinge einschob, welche die Veterinärchirurgie betreffen». Unter anderem verweist er auf dessen Methode der Blutstillung bei der Exstirpation einer geschwollenen Drüse am Pferd: Schon Crescentius habe nämlich empfohlen, falls es bei der Excision zu einer stärkeren sanguinatio kommen sollte, das blutende Gefäß zu fassen und mit einem seidenen Faden fest zusammenzuschnüren. Der Paduaner liebt rhetorische Fragen, wenn er eigene Ansichten besonders hervorheben will, und so fragt er auch hier: «Glauben Sie etwa, er (Crescentius) habe dies nicht aus älteren Tierärzten geschöpft? Diese aber nicht aus anderen, die noch bei weitem älter waren? Vielleicht gar war es jenen schon geläufig, noch bevor CeLsus und GaLEN die Blutstillung an verwundeten Körperteilen durch Fadenligatur der verletzten Vene oder Arterie lehrten, sei es nun, daß sie eine Drüse exstirpierten oder Pferde behandelten, die im Kampf verletzt worden waren.» Mit den folgenden Sätzen kehrt Morgagni endgültig zur Humanchirurgie zurück: Denn ob nun diese Art der Blutstillung auf den Menschen übertragen, oder an ihm zum erstenmal angewandt wurde, so sei sie doch seit Galens Zeiten hier und da in den Büchern der Chirurgen beschrieben und unter den älteren Wundärzten von BENIVIENI bei einer Penisamputation auch praktisch ausgeführt worden. So wundere man sich unwillkürlich, «daß niemand vor Paré aufgetreten ist, der auf den Gedanken kam, jene Methode könnte auch auf die Wunden übertragen werden, die bei den Gliedmaßenamputationen entstehen».

Und um mögliche Einwände vorwegzunehmen, beschließt er seine Ausführungen mit den Worten: «Doch glauben Sie nicht etwa, man hätte sich 
vor der Dicke der Arterien gefürchtet, die in den Gliedmassen die Hauptstämme bilden, da diese sich mit der Feinheit der Äste, die Galen zu unterbinden vorschrieb, nur schwerlich vergleichen lassen. Jedenfalls ist der Hauptstamm am Arm zugleich jener, den unerfahrene Menschen häufig in der Ellenbeuge verletzen in der Meinung, eine Vene zu öffnen, woraus später ein Aneurysma hervorgeht, wenn man nicht gleich am Anfang sorgfältige Gegenmaßnahmen trifft. Und dennoch hätte man nicht nur aus den Schriften des Aetius, des Paulus, des Abulkasis, sondern auch aus denen weniger bedeutender Ärzte, wie des GuY DE Chauliac sehr wohl wissen können, daß gerade der genannte Hauptast bei der Behandlung dieses Aneurysma mit Fäden unterbunden zu werden pflegt».

Auch hier versagt sich der Paduaner ein Wort der Anerkennung, er bleibt knapp und nüchtern, und wie an anderen Stellen vermeidet er einen Hinweis in den Registern. Dennoch hält er seine Sätze von jenen bösen und kurzsichtigen Anwürfen frei, mit denen um der Gefäßligatur willen einst Etienne Gourmelen, der Dekan der Pariser medizinischen Fakultät, Paré zu Unrecht überhäuft hatte ${ }^{52} . \mathrm{Da} \beta$ andererseits die Erfindung der Gefäßunterbindung bei der Gliedmaßenamputation, wenn man sie lediglich als den Endpunkt einer langen Entwicklung betrachtete, erheblich an Verdienst einbüßen mußte, dürfte Morgagni freilich nur willkommen gewesen sein. Allein Paré hat seine eigene Leistung in dieser Sache nicht anders gesehen, wie uns seine Apologie belehrt ${ }^{53}$, und eine Überprüfung der zitierten Ärzte zeigt, daß Morgagni hier tatsächlich nur Parés historischen Standort bestimmte. Denn Celsus befürwortet die Literatur an mehreren Stellen seines Werkes ${ }^{54}$, und für Galens «Therapeutische Methode» ist ihre Empfehlung in bestimmten, allerdings eng begrenzten Fällen bekannt ${ }^{55}$. Auch

52 Vgl. E.H. ACKerKNecht, in Hubers Klassiker der Medizin und der Naturwissenschaften, Band II, Bern/Stuttgart 1963, S. 7 und 14 f.

${ }^{53}$ Vgl. Hubers Klassiker der Medizin und der Naturwissenschaften, Band II, S. 17. Als Vorgänger für die Gefäßunterbindung zählt PARÉ hier folgende Autoritäten auf: Galen, Avicenna, Guy de Chauliac, Hollier, Calmetheus, Celsus, Vesal, Tagault, Petrus von Argilata, Johann Andreas à Cruce und d'Alechamp. Auch in seinen Opera chirurgica (ibidem) sagt er unter Berufung auf Galen: «Hoc Galeni praeceptum de vinciendis et suendis venis et arteriis, in vulneribus recentibus, ad ea quae membrorum amputatione fiunt cum traduci posse putarem, tentavi in plerisque ...»

${ }^{54}$ Celsus, De medicina, lib. V, c. 26, 21 C; Ed.Loeb II, p. 80. Neben dieser von Morgagni zitierten Stelle siehe auch lib. VII, c. 19, 3.5.8 und c. 22, 2.5; Ed.Loeb III, p. $400 \mathrm{ff}$. und $416 \mathrm{f}$.

${ }^{55}$ Galen, De methodo medendi, lib. V, c. 3; X 319 K. Sowohl Paré als auch Morgagni berufen sich auf diese Stelle, MorgagNI schreibt allerdings lib. IV statt V. 
Aetios und Paulos von Aegina haben sie geübt ${ }^{56}$, und Abulkasim und Guy de Chauliac stehen in derselben Tradition ${ }^{57}$. Selbst dem Beispiel Benivienis lassen sich unschwer weitere Autoren an die Seite stellen, die bezeugen, daß die Gefäßunterbindung auch in der Praxis immer wieder angewandt wurde ${ }^{58}$. So stellt auch die Aufzählung in Parés Apologie nur eine höchst unvollständige Auswahl dar, und nicht nur Henri de Mondeville blieb hier unberücksichtigt, den weder Paré noch Morgagni gekannt haben ${ }^{59}$.

Kaum weniger gewichtig aber dürfte Morgagnis Hinweis auf die Tiermedizin sein, darf er doch gerade für die älteren Werke über Agrikultur und Tierheilkunde als hervorragender Kenner gelten. Denn einmal hat er in eigenen Briefen die Schriften des Varro und Columella kommentiert ${ }^{60}$, zum anderen aber hat er über seinen Schüler TARGA die moderne CelsusForschung angeregt. Auch er selbst hat sich sein Leben lang mit Celsus beschäftigt, dessen verlorene fünf Bücher über die Landwirtschaft die Grundlage zu den Schriften des Columella und Varro bildeten ${ }^{61}$. Mag er sich

56 Aetios, Tetrabibl. IV, serm. 2, c. 40; Ed. J.Cornarius, Basel 1549, p. 771 (in Morgagnis Zitatnachweis fälschlicherweise: serm. 4 statt serm. 2). Die hier geschilderte Varizenoperation erwähnt die Gefäßligatur zwar nicht ausdrücklich, dürfte sie aber stillschweigend voraussetzen. - Paulus Aegin., Opus de re medica, lib. VI, c. 37; Ed. Venedig 1542, p. $230 \mathrm{f} .=\mathrm{cmG} \mathrm{IX} \mathrm{2,} \mathrm{p.} 77$ (Aneurysmenoperation).

57 Abulcasim, Methodus medendi certa, etc., Basel 1541 (lateinische Übersetzung), P. II, c. 49 ; p. 95. Diese von Morgagni zitierte Stelle enthält einen Verweis auf P. II, c. 3; p. 47, wo das technische Verfahren für die Arterienligatur angegeben ist. Bei speziellen Eingriffen empfohlen findet sie sich außerdem z.B.: P. I, c. 57 ; p. 42. - P. II, c. 81; p. 135. - P. II, c. 84; p. 139. - GUY DE Chauliac, Chirurgia magna, Ed. Lyon 1585, Tr. II, Dec. II, c. 4; p. 101 (Aneurysmenoperation).

58 A. Benivieni, De abditis nonnullis ac mirandis morborum et sanationum causis, Florenz 1507 (unpaginiert), c. 68. - So teilt z.B. Rrverius eine Gefäßligatur nach Oberarmverletzung in Axialnähe als Observ. II des Samuel Formio, Chirurgiae Magister zu Montpellier, mit (Opera medica universa, Lyon 1698, p. 570).

59 Bei Henri de Mondeville findet sich die Gefäßligatur sogar bereits bei der Gliedmaßenamputation. Vgl. J.L.PAgeL, Die Chirurgie des Heinrich von Mondeville, Arch. klin. Chir. 40 (1890) 877, 881 und $884=$ Tract. II, Doctr. I, c. 1, pars 2 (Wundnaht bei stark blutenden Wunden) und Arch. klin. Chir. 41 (1891) $196=$ Tract. III, Doctr. I, c. 6 (Gefäßligatur bei Amputation). - Siehe auch E. GurLt, Geschichte der Chirurgie, Band II, Berlin 1898, S. 48 f. (Wundnaht) und S. 67 (Amputation) und P. HuARd et M. D. GrMEK, Mille ans de Chirurgie, Paris 1966, p. 40.

${ }^{60}$ Vgl. Verfasser, Hubers Klassiker der Medizin und der Naturwissenschaften, Band X, Bern/Stuttgart 1967, S. $17 \mathrm{f}$.

${ }^{61}$ Zum Verhältnis zwischen Celsus einerseits und Columella und VARRo andererseits vgl. J. Ilberg, A. Cornelius Celsus und die Medizin in Rom, Neue Jahrbücher für das klassische Altertum, Geschichte und deutsche Literatur 19, Jahrgang 10 (1907) $379 \mathrm{ff}$. 
daher auch für die Drüsenexstirpation beim Pferd auf Crescentius beziehen, dessen Beschreibung die Gefäßligatur nicht ausdrücklich vermerkt ${ }^{62}$, so setzt das Gelingen des Eingriffs doch ähnlich wie die Venenresektion bei Aetios die Unterbindung voraus, und tatsächlich beschreibt sie in diesem Zusammenhang auch Jordanus Ruffus, den Crescentius nur ausgeschrieben hat ${ }^{63}$. So dürfte auch Morgagnis Vermutung, Crescentius habe seine chirurgischen Praktiken von älteren Tierärzten übernommen, der Wahrheit sehr nahe kommen; denn wir wissen heute, daß er beziehungsweise Ruffus aus Griechen und Lateinern, Byzantinern und Arabern geschöpft hat ${ }^{64}$. Für die Tatsache aber, daß auch die spätantike Tiermedizin die Gefäßligatur gekannt hat, läßt sich die «Mulomedicina Chironis» als eindeutiges Beispiel nennen ${ }^{65}$, und selbst Morgagnis Hinweis auf alte mündliche Überlieferungen innerhalb der Human- und Veterinärchirurgie weiß die moderne medizinhistorische Forschung gebührend zu würdigen ${ }^{66}$.

Nur wer sich von den Ergebnissen der vorangehenden Untersuchungen allzusehr gefangennehmen ließe, könnte daher zu der Ansicht neigen, auch diese letzte Diskussion habe es mit dubiösen Argumenten allein auf eine Schmälerung von Parés Verdiensten abgesehen. Eine sachliche Überprüfung zeigt, daß auch Paré, wie jeder noch so bedeutende Meister, seinen Vorgängern verpflichtet bleibt, und es braucht ihm gewiß nichts von seiner Größe zu nehmen, wenn sich hier eher die Feststellung treffen läßt, daß die

62 Petrus de Crescentirs, De omnibus agriculturae partibus et de plantarum animaliumque natura et utilitate, lib. IX, c. 16; Ed. Basel 1542, p. 277 f. Der Text stimmt bis auf unbedeutende Wortabweichungen mit der Ed.: Opus ruralium commodorum 1474 überein, die verglichen werden konnte, und auch die älteste deutsche Übersetzung erwähnt in den einschlägigen operativen Kapiteln (cc. 52-55) nirgends ausdrücklich die Gefäßligatur (vgl. O. Bederke, Liber de cura equorum, Bearbeitungen von Albertus MAGNus und Jordanus Ruffus aus dem deutschen Ritterorden, Hannover 1962 (Diss. med. vet.), S. $46 \mathrm{f}$.

${ }^{63}$ Vgl. den Hinweis bei F. Eichbaum, Grundriß der Geschichte der Tierheilkunde, Berlin 1885, S. 43. - Eine Ausgabe des Jordanus RufFus war mir leider nicht zugänglich.

${ }^{64}$ Gegen F. Eichbaum (loc. cit.) siehe E. Leclainche, Histoire illustrée de la médecine vétérinaire, presentée par G. RAmoN, Tom. I, Monaco 1955, p. 182 ; vgl. auch O. BEDERKE, a.a. O., S. 11.

65 Vgl. M. Niedermann, Proben aus der sogenannten Mulomedicina Chironis (Buch II und III), Sammlung vulgärlateinischer Texte, Band 3, Heidelberg 1910, lib. II, c. 20; p. 11 f.

${ }^{66}$ Zur Frage der mündlichen Überlieferung in der Chirurgie vgl. H.E.Sigerist, Anfänge der Medizin, Zürich 1963, S. 22. Ähnlich urteilt P. DiePGen über die mündliche Tradition unter den Hebammen; vgl. Frau und Frauenheilkunde in der Kultur des Mittelalters, Stuttgart 1963, S. 15 f. 
Geschichte der Gefäßligatur einer neuen Bearbeitung bedarf, die auch die Veterinärchirurgie der älteren Zeiten in ihre Betrachtung einzubeziehen hätte. Morgagni, mit der Erfahrung eines langen Lebens und umfassenden, weit in die Vergangenheit zurückreichenden Literaturkenntnissen begabt, scheint schon damals das rechte Augenmaß in dieser Frage besessen zu haben.

So bleibt es auch in diesem Abschnitt bei der unauffälligen Abwertung mit protokollarischen Mitteln, Paré bleibt auch hier Paré, dem sich nichts zugute halten läßt. Morgagni aber muß mit dieser seiner Gesinnung im Rahmen der Zeit gesehen werden, er hielt sich in seinem Werk offenbar an ein ungeschriebenes Gesetz, das unverminderte Gültigkeit besaß: Noch immer waren die alten nicht-akademischen Chirurgen von einst schlecht angesehen, ihre Entdeckungen und Praktiken waren zwar übernommen worden und ihre Observationes wurden gebraucht, sie selbst aber blieben noch immer ausgesperrt aus der Tradition der akademischen Medizin. Erst mit der endgültigen Eingliederung der Chirurgie in die Universitätsmedizin wurde dieser Frage die Animosität genommen und der Weg freigemacht für eine sachliche Würdigung ihrer Leistungen. 